\title{
Crescentic Glomerulonephritis with Positive Antineutrophil Cytoplasmic Autoantibody Specific for Myeloperoxidase Associated with Autoimmune Hemolytic Anemia and Thrombocytopenic Purpura
}

\author{
Junichi OHhashi, Masaki MiYamoto, Shinsuke Ishikawa, Youichi Hashizume, Takaaki Nishido, \\ Kimihiko Matsuyama, Kazuhide Morino* and Yasushi MiYazaki
}

\begin{abstract}
A 41-year-old woman was admitted to the hospital with severe uremia, hemolytic anemia, and thrombocytopenic purpura. Emergency hemodialysis with plasmapheresis was started in view of consideration of hemolytic uremic syndrome (HUS), which resulted in improvement of renal function and platelet count. Positive antineutrophil cytoplasmic autoantibody specific for myeloperoxidase (MPOANCA) suggested crescentic glomerulonephritis, which was pathologically evidenced by renal biopsy. The diagnosis of MPO-ANCA associated crescentic glomerulonephritis with autoimmune hemolytic anemia (AIHA) and thrombocytopenic purpura were comfirmed. Three courses of steroid pulse therapy with heparin were successfully performed, followed by oral prednisolone and warfarin. Such a case has not been previously reported to our knowledge. (Internal Medicine 39: 650-654, 2000)
\end{abstract}

Key words: RPGN, MPO-ANCA, HUS, Evans syndrome

\section{Introduction}

Antineutrophil cytoplasmic autoantibodies have been known to be closely related to microscopic vasculitis, including ChurgStrauss syndrome and Wegener's granulomatosis, rapidly progressive glomerulonephritis (RPGN), pulmonary hemorrhage, and also concurrent features of two or more of these diseases (1).

For editorial comment, see $\mathrm{p} 603$.

In recent years, antineutrophil cytoplasmic autoantibody specific for myeloperoxidase (MPO-ANCA) associated glomerulonephritis has been noticed, especially in Japan, because of characteristic features clinically presenting RPGN and pathologically, pauci-immune crescentic necrotizing glomerulonephritis (2-4).

We treated a case of MPO-ANCA positive crescentic glomerulonephritis associated with autoimmune hemolytic anemia (AIHA) and thrombocytopenic purpura with elevated plateletassociated immunoglobulin G (PA-IgG). No such case has been previously reported to our knowledge.

\section{Case Report}

A 41-year-old woman was admitted to our hospital because of tiredness, nausea and petechiae. She had been well until two weeks before admission, when she had flu-like symptoms, followed by fever. She consulted a doctor on March 30, 1998 and became afebril. On April 4 she noticed oral bleeding and visited an out-patient clinic, where the laboratory examination revealed serious renal dysfunction (serum creatinine $11.68 \mathrm{mg} /$ $\mathrm{dl}$ ), anemia (hemoglobin $5.2 \mathrm{~g} / \mathrm{dl}$ ) and thrombocytopenia (platelet count $\left.2.3 \times 10^{4} / \mu \mathrm{l}\right)$. She had no history of renal disease and a urinalysis was normal in 4 years previously. She was transferred to our hospital on April 7, 1998.

On admission, blood pressure was $140 / 90 \mathrm{mmHg}$, pulse was regular at $82 / \mathrm{min}$, respirations were $24 / \mathrm{min}$, and temperature was $36.4^{\circ} \mathrm{C}$. The patient was alert, but looked pale and severely ill. Mucosal bleeding of mouth and petechiae on the abdominal wall and forearms were found. Physical examination of the chest was normal. Auscultation and palpation of the abdomen were normal. The neurologic examination revealed no abnormal findings.

The plain chest X-ray film and the electrocardiogram were normal. On abdominal sonogram, the kidneys seemed slightly decreased in size, but not significant. Laboratory examination,

From the Department of Internal Medicine, Misato Kenwa Hospital, Misato and *Waseda Clinic, Misato

Received for publication July 16, 1999; Accepted for publication February 21, 2000

Reprint requests should be addressed to Dr. Junichi Ohhashi, the Department of Internal Medicine, Misato Kenwa Hospital, 4-494-1 Takano, Misato, Saitama $341-8555$ 
RPGN with MPO-ANCA, AIHA and Purpura

Table 1. Laboratory Data on Admission

\begin{tabular}{|c|c|c|c|}
\hline \multirow{2}{*}{$\begin{array}{l}\text { Urinalysis } \\
\text { Glucose }\end{array}$} & \multicolumn{2}{|r|}{ Coagulation profiles } & \\
\hline & $(-)$ & Prothrombin time & $11.8 \sec (11.3)$ \\
\hline Protein & $(+++)$ & Activated partial thromboplastin time & $29.1 \mathrm{sec}$ \\
\hline Ketone body & $(-)$ & Fibrinogen & $481 \mathrm{mg} / \mathrm{dl}$ \\
\hline Occult blood & $(+++)$ & FDP & $20.0 \mu \mathrm{g} / \mathrm{ml}$ \\
\hline Casts & & Antithrombin III & $120 \%$ \\
\hline Hyaline cast & $(+)$ & Immunological examination & \\
\hline Granular cast & $(+)$ & C-reactive protein & $1.0 \mathrm{mg} / \mathrm{dl}$ \\
\hline Epithelial cast & $(+)$ & Haptoglobin & $<12 \mathrm{mg} / \mathrm{dl}$ \\
\hline Hematological examination & & Antinuclear antibody & $(-)$ \\
\hline White blood cells & $7,500 / \mathrm{mm}^{3}$ & Anti-DNA antibody & $3.0 \mathrm{IU} / \mathrm{ml}$ \\
\hline Red blood cells & $118 \times 10^{4} / \mathrm{mm}^{3}$ & Complement activity & $46.9 \mathrm{CH}_{50} / \mathrm{ml}$ \\
\hline Hemoglobin & $3.6 \mathrm{~g} / \mathrm{dl}$ & $\mathrm{C} 3$ & $77 \mathrm{mg} / \mathrm{dl}$ \\
\hline Hematocrit & $10.5 \%$ & $\mathrm{C} 4$ & $36 \mathrm{mg} / \mathrm{dl}$ \\
\hline Platelet & $5.0 \times 10^{4} / \mathrm{mm}^{3}$ & Immunecomplex & $<1.5 \mu \mathrm{g} / \mathrm{ml}$ \\
\hline Reticulocytes & $3.6 \%$ & Immunoglobulin $\mathrm{G}$ & $1,900 \mathrm{mg} / \mathrm{dl}$ \\
\hline Blood chemistry test & & Immunoglobulin $\mathrm{M}$ & $151 \mathrm{mg} / \mathrm{dl}$ \\
\hline Aspartate aminotransferase & $38 \mathrm{U} / \mathrm{l}$ & Immunoglobulin A & $256 \mathrm{mg} / \mathrm{dl}$ \\
\hline Alanine aminotransferase & $22 \mathrm{U} / l$ & Direct Coombs' test & $(+)$ \\
\hline Alkaline phosphatase & $216 \mathrm{U} / l$ & Indirect Coombs' test & $(-)$ \\
\hline Lactate dehydrogenase & $905 \mathrm{U} / l$ & MPO-ANCA & $171 \mathrm{EU}$ \\
\hline$\gamma$-glutamyltranspeptitase & $40 \mathrm{U} / l$ & PR3-ANCA & $<10 \mathrm{EU}$ \\
\hline Total bilirubin & $0.33 \mathrm{mg} / \mathrm{dl}$ & Anti-GBM antibody & $<5 \mathrm{EU}$ \\
\hline Direct bilirubin & $0.10 \mathrm{mg} / \mathrm{dl}$ & PA-IgG & $43.8 \mathrm{ng} / 10^{7}$ cells \\
\hline Total Protein & $6.5 \mathrm{~g} / \mathrm{dl}$ & Blood gas analysis (room air) & \\
\hline Albumin & $3.3 \mathrm{~g} / \mathrm{dl}$ & $\mathrm{pH}$ & 7.390 \\
\hline Blood urea nitrogen & $120.4 \mathrm{mg} / \mathrm{dl}$ & $\mathrm{PaCO}_{2}$ & $29.8 \mathrm{mmHg}$ \\
\hline Creatinine & $12.2 \mathrm{mg} / \mathrm{dl}$ & $\mathrm{PaO}_{2}$ & $105.6 \mathrm{mmHg}$ \\
\hline $\mathrm{Na}$ & $131 \mathrm{mEq} / \mathrm{l}$ & $\mathrm{HCO}_{3}^{-}$ & $18.0 \mathrm{mmol} / \mathrm{l}$ \\
\hline $\mathrm{K}$ & $5.2 \mathrm{mEq} / \mathrm{l}$ & Base excess & $-5.9 \mathrm{mmol} / \mathrm{l}$ \\
\hline $\mathrm{Cl}$ & $94 \mathrm{mEq} / \mathrm{l}$ & & \\
\hline $\mathrm{Fe}$ & $130 \mu \mathrm{mg} / \mathrm{dl}$ & & \\
\hline
\end{tabular}

FDP: Fibrin/Fibrinogen degradation products, $\mathrm{C} 4$ : The fourth component of complement, $\mathrm{PaCO}_{2}$ : Partial pressure of carbon dioxide, $\mathrm{PaO}_{2}$ : Partial pressure of oxygen.

as shown in Table 1, revealed severe uremia, anemia, and thrombocytopenia. The urine was positive for protein $(+++)$, occult blood (+++), and cellular casts (+). Hemoglobin was $3.6 \mathrm{~g} / \mathrm{dl}$, hematocrit was $10.5 \%$, with $3.6 \%$ reticulocytes, and the platelet count was $5.0 \times 10^{4} / \mathrm{mm}^{3}$. Serum creatinine was $12.2 \mathrm{mg} / \mathrm{dl}$ and blood urea nitrogen was $120.4 \mathrm{mg} / \mathrm{dl}$, with mild metabolic acidosis. Elevated lactate dehydrogenase was noticed with normal total bilirubin and aspartate aminotransferase. Coagulation study was normal.

Immunological examination was negative for anti-nuclear antibody (ANA), anti-DNA antibody, anti-glomerular basement membrane antibody (anti-GBM antibody) and immunecomplex, and positive for MPO-ANCA and direct Coombs' test with marked decrease in haptoglobin. A mild increase in PA-IgG was noticed.

The sternal bone marrow aspiration specimen showed mild hyperplastic marrow with erythroid-hyperplasia and increased megakaryocytes without excess of blastic cells. Helmet cells were found in the peripheral blood smear. The clinical course of the patient is shown in Fig. 1.
Immediately after admission, when the information of immunological examination except for positive direct Coombs' test, had not been obtained, acute renal failure possibly due to hemolytic uremic syndrome (HUS) was highly suspected. Hemodialysis was started on the second hospital day, with two courses of plasmapheresis and two units of washed red blood cells transfusion on the third and fourth hospital days. In total, five courses of hemodialysis with additional two units of washed red blood cells transfusion were performed with sufficient clinical improvement. Serum creatinine was $4.4 \mathrm{mg} / \mathrm{dl}$, hemoglobin was $8.1 \mathrm{~g} / \mathrm{dl}$, and platelet count was $17.7 \times 10^{4} / \mathrm{mm}^{3}$ on the 10th hospital day. On the other hand, the information of immunological examination on admission had been obtained on the 9th hospital day, which revealed a strong positive test for MPO-ANCA. The immunological data were highly suggestive of MPO-ANCA associated RPGN, with manifestation of HUS.

From the 10th hospital day, the first steroid pulse therapy, consisting of intravenous methylprednisolone $(1,000 \mathrm{mg}$ daily dose) was administered for three days. Thereafter the patient 
was in stable condition, without additional medication, such as oral prednisolone. On the 21st hospital day, surgical renal biopsy was performed.

Pathological findings of the biopsied specimen containing about 200 glomeruli on a section, revealed marked crescent formation, frequently surrounding the glomerular tuft, in over $90 \%$ of the glomeruli. And most of the glomeruli with crescent formation showed a marked increase of the mesangial matrix and cells, subsequently presenting the features of global ne- crosis (Fig. 2). In the interstitium, marked mononuclear cell infiltration was found, partially showing granulomatous change. Mononuclear cell infiltration was also observed in the perivascular space and intramurally in the small vessels, being suggestive of vasculitis (Fig. 3). Immunofluorescent staining for immunogloblin and complement was positive only for immunogloblin $\mathrm{M}$ and the third component of complement (C3). Electron microscopic examination revealed mesangial and subendothelial electron dense deposits with mesangial in-

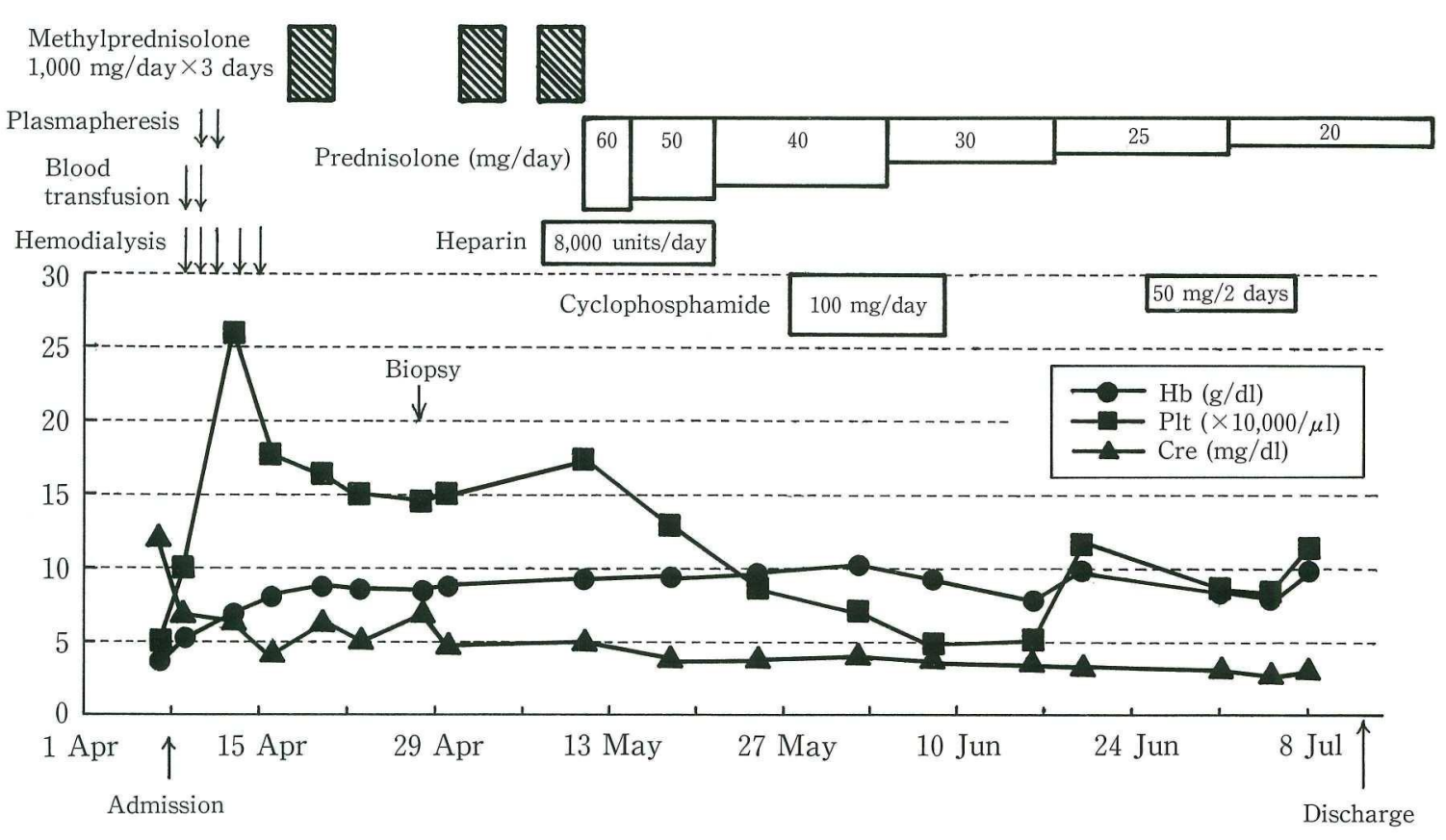

MPO-ANCA 4/8 171 EU

$6 / 219 \mathrm{EU}$

$7 / 37 \mathrm{EU}$

Figure 1. Clinical course.

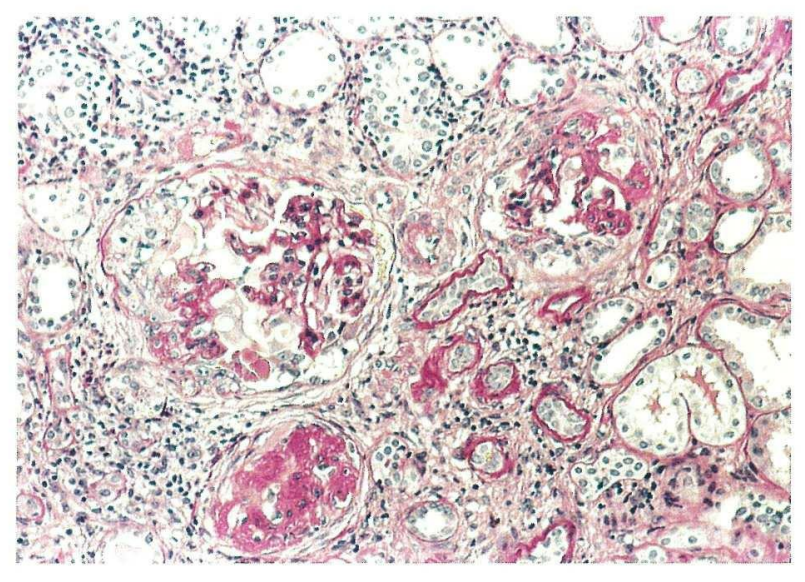

Figure 2. Crescentic glomerlonephritis with marked lymphocytic infiltration in the interstitium (PAS stain, $\times 200$ ).

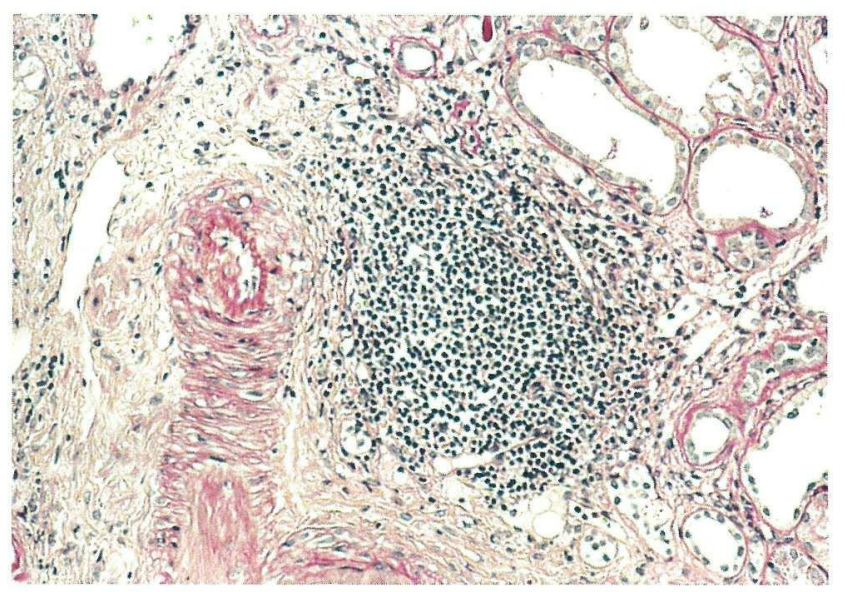

Figure 3. Granulomatous change (PAS stain, $\times 400)$. 


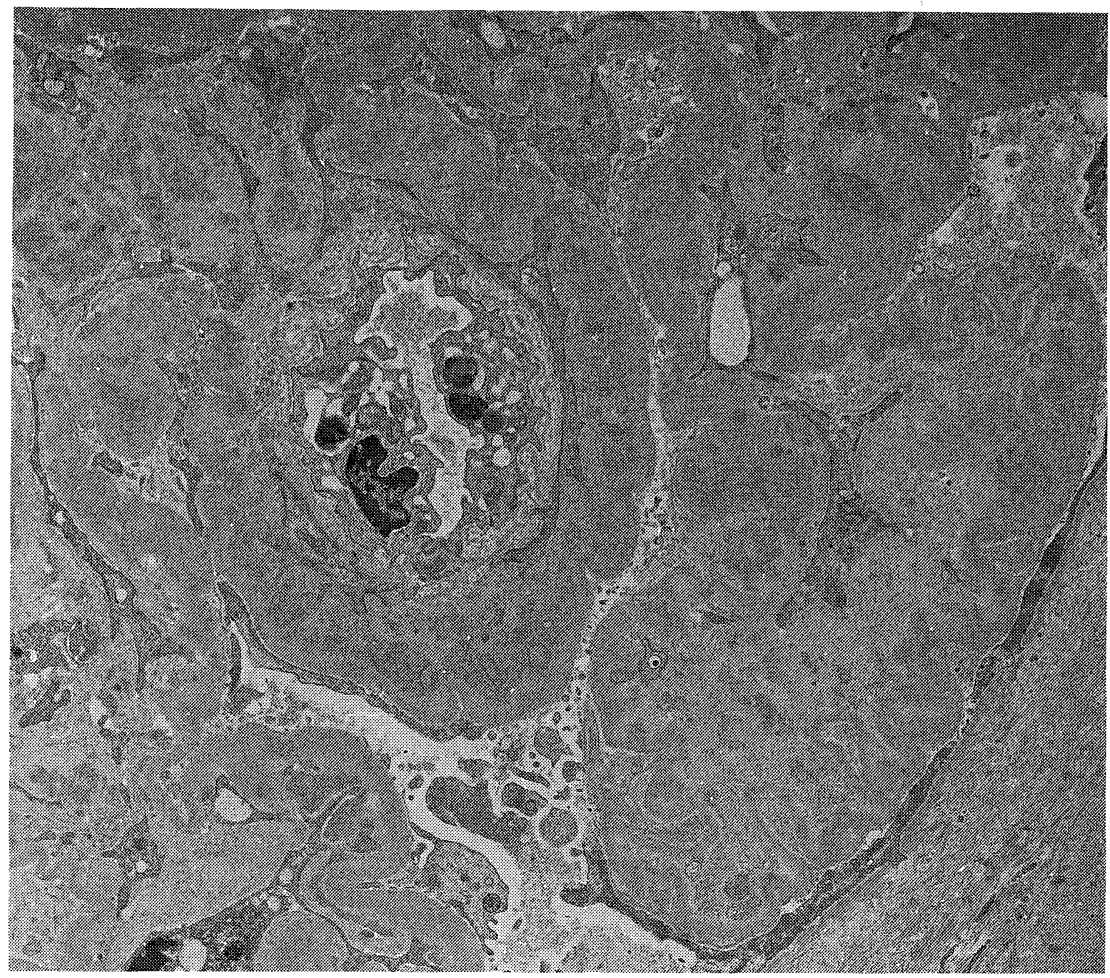

Figure 4. Mesangial and subendothelial electron dense deposits with mesangial interposition (Electronmicroscopic examination, $\times 2,500)$.

terposition (Fig. 4).

Diagnosis of MPO-ANCA positive crescentic glomerulonephritis with AIHA and PA-IgG positive thrombocytopenic purpura was confirmed. From five days after the renal biopsy, the second course of pulse therapy was performed without improvement of the serum creatinine level, anemia and the finding of urinalysis, as compared with those after the first pulse therapy. One week later, when the diagnosis was established, the third course of pulse therapy was performed with concurrent use of heparin ( 8,000 units/day by drip infusion) for two weeks, followed by oral administration of prednisolone $(60 \mathrm{mg} /$ day) after the pulse therapy and warfarin ( $2 \mathrm{mg} /$ day) substituted for heparin two weeks later. Additional use of cyclophosphamide was given up because of the sudden occurrence of leukocytopenia. Renal dysfunction had gradually improved, and the dose of prednisolone was gradually reduced. The serum creatinine level was $3.3 \mathrm{mg} / \mathrm{dl}$ with negative result for MPOANCA and direct Coombs' test, and prednisolone was given at a daily dose of $20 \mathrm{mg}$ from the time of discharge on July 13 , 1998.

\section{Discussion}

The acutely ill patient characterized by severe anemia, thrombocytopenia and renal failure on admission suggested some kind of diseases such as HUS, thrombotic thrombocytopenic purpura, lupus nephritis with Evans syndrome and less pos- sible hematologic malignancies. The lack of neurological symptoms, normal coagulation study and bone marrow examination showing erythro-magakaryocytic hyperplasia without excess of myeloblasts, highly supported the presence of HUS. In the early days after admission, the clinical features were compatible with HUS. Accordingly, emergency hemodialysis including plasmapheresis was performed, resulting in sufficient clinical improvement.

Immunological data obtained a week later, revealed positive MPO-ANCA, negative ANA, positive Coombs' test and elevated PA-IgG level. These immunological findings, considering a probable subacute clinical course, strongly suggested MPO-ANCA associated crescentic glomerulonephritis, clinically presenting RPGN, accompanied with AIHA and PA-IgG positive thrombocytopenic purpura.

The final diagnosis was confirmed by the histopathology of renal biopsy, with severe crescentic glomerulonephritis, interstitial nephritis, and microscopic vasculitis. But unexpectedly some problems were raised, that the findings of electron dense deposits and mesangial interpositions are observed in electronmicroscopic examination are incompatible with those commonly described in MPO-ANCA associated glomerulonephritis. The presence of preceding renal disease, such as membranoproliferative glomerulonephritis where electron dense deposit and mesangial interposition are commonly demonstrated, may be less possible, because of the lack of history of renal disease and abnormal urinalysis. It remains unknown whether the elec- 


\section{OHHASHI et al}

tron dense deposits may be immune-complex or not.

The clinical features of HUS are characterized by non-AIHA, thrombocytopenia by intravascular consumption and acute renal failure, pathologically showing thrombotic microangiopathy. Although the clinical manifestations of the present case are compatible with those of HUS, the presence of Coombs' antibody and elevated PA-IgG suggests more likely AIHA and thrombocytopenia. In a recent report, MPO-ANCA positive crescentic glomerulonephritis presenting HUS was described, where deposits of fluffy and fibrillar material were observed in the vascular subendothelial space by electronmicroscope (5). The electron dense deposit of the present case seems different from that of the reported case. An additional case with antineutrophil cytoplasmic autoantibody specific for proteinase 3 (PR3-ANCA or c-ANCA) related RPGN presenting HUS also did not have autoantibodies, except for c-ANCA (6).

From the above-mentioned viewpoint, we considered the present case to be a rare one with the coincidence of MPOANCA positive crescentic glomerulonephritis, AIHA, and thrombocytopenic purpura, presenting HUS-like clinical features.

Retrospectively, plasmapheresis was very effective for preventing progress of renal failure, improvement of anemia and thrombocytopenia. Plasmapheresis was also effective in the reported cases. The success of the initial treatment produced good results in the following treatment with corticosteroid pulse and anticoagulant therapy. The usual treatment for RPGN is combination therapy with corticosteroid and cyclophosphamide. In this case cyclophosphamide was discontinued because of the emergence of leukocytopenia soon after administration. Ultimately, the patient unfortunately died of systemic nocardiosis 8 months later.

As for the prognosis, a study by the Ministry of Health and Welfare revealed that MPO-ANCA was positive in $64 \%$ of 583 cases with RPGN, $31.5 \%$ received hemodialysis, and $21.4 \%$ of patients died within 6 months after the start of the treatment (7).

\section{References}

1) Jennete JC, Folk RJ. Antineutrophil cytoplasmic autoantibodies and associated disease: a review. Am J Kidney Dis 15: 517-529, 1990.

2) Tanemoto M, Miyakawa H, Hanai J, Yago M, Kitaoka M, Uchida S. Myeloperoxidase-antineutrophil cytoplasmic antibody-positive crescentic glomerulonephritis complicating the course of Graves'disease: report of three adult cases. Am J Kidney Dis 26: 774-780, 1995.

3) Kudoh Y, Kuroda S, Shimamoto K, Iimura O. Propylthiouracil-induced rapidly progressive glomerulonephritis associated with antineutrophil cytoplasmic autoantibodies. Clin Nephrol 48: 41-43, 1997.

4) Pai P, Rustom R, Bone JM, Bell GM. Chronic bronchiectasis and antimyeloperoxidase antibody related rapidly progressive necrotizing glomerulonephritis. Clin Nephrol 49: 262-264, 1998.

5) Hirsch DJ, Jindal KK, Trillo AA. Antineutrophil Cytoplasmic AntibodyPositive Crescentic Glomerulonephritis and Thrombotic Microangiopathy. Am J Kidney D 26: 385-386, 1995.

6) Stefanidis I, Helmchen U, Schmitt H, Maurin N, Sieberth HG. Coincidence of haemolytic uraemic syndrome and c-ANCA-associated rapidly progressive glomerulonephritis. Nephrol Dial Transplant 13: 1818-1821, 1998.

7) Yamaguchi N, Muro K, Kikuchi S, Kobayashi M, Takahashi H, Koyama T. Rapidly progressive glomerulonephritis. J Jpn Soc Int Med 87: 12541262, 1998 (in Japanese). 